\title{
MEASUREMENT OF ECCENTRIC FIXATION BY THE BJERRUM SCREEN*
}

\author{
BY \\ G. BROCKBANK AND R. DOWNEY \\ General Infirmary, Leeds
}

Introduction by G. W. Black and J. Foster.-The forward movement of orthoptic technique between 1925 and 1935 depended on a re-appreciation of the importance of anomalous retinal correspondence, measured by the improved synoptophores then available. Before this, many patients had been wasting their time on unsupervised divergence exercises.

In this decade Cüppers's discovery of the high percentage of amblyopic eyes with eccentric fixation which are detectable by the Visuscope and undetectable by the synoptophore, is likely to have a similar effect on the occlusion treatment of amblyopia.

The collaboration of Miss R. Downey (Orthoptist) and Mr. G: Brockbank (Ophthalmic Optician) at the Leeds General Infirmary has produced a subjective method which will (when applicable) detect an even smaller degree of eccentric fixation, and is, we think, of importance in view of the enormous effort that pleoptic treatment requires both from patient and therapist.

\section{Eccentric Fixation}

The pleoptic treatment of deep amblyopia, especially that of eccentric fixation, was introduced into Great Britain nearly 2 years ago. The methods of treatment were described first by Dr. A. Bangerter of St. Gallen, Switzerland, and later by Dr. C. Cüppers of Giessen, Germany. Strictly speaking, the word "pleoptics" referred to Bangerter's method rather than that of Cüppers, which was entirely different in its physiological conception. These two methods are at present the subject of experiment in London, Manchester, and Leeds, but very few statistics are yet available.

At Leeds we have treated eccentric fixation experimentally by Cüppers's method, the instruments principally used were the Visuscope, the Euthyscope, and the Co-ordinator.

The Visuscope is an ophthalmoscope in which there is, amongst other discs, one containing a black star. This can be focused onto the fundus by allowing for the patient's refractive error, and when the patient is fixing the star, the point where it appears on the retina indicates to the observer the principal direction of gaze of that eye. During an examination with the Visuscope it is necessary to occlude the non-fixing eye to avoid the influence of anomalous retinal correspondence. 
The Euthyscope is used to create a negative after-image, which is an essential part of Cüppers's treatment of eccentric fixation and inhibition. This instrument is a large ophthalmoscope with a special system of lenses permitting a high illumination of approximately $30^{\circ}$ of the retina. Either of two central black spots may be selected to allow protection of $5^{\circ}$ or $3^{\circ}$ of the retina. An enormous ring-shaped after-image is thus formed by illuminating the posterior pole of the eye, the macula being protected by one of the black spots. A positive after-image, it will be appreciated, still maintains the amblyopic state; so a negative after-image is sought (a dark ring with clear centre) by projecting it on to an illuminated screen from 1 to 6 metres distant. The patient's attention is drawn to the central disc of this negative after-image which is a real object in space characterizing the fovea.

The Co-ordinator uses Haidinger's brushes as a subjective control for macular fixation. Its basis is an entoptic phenomenon recognized when plane or moderately elliptically polarized light enters the normal eye, and depends entirely upon regional variations in the brightness of the blue component of the incident light. The two brush-like objects seen are the visual residue of the absorption of the polarized component of the sky light by the double-refracting yellow lutein pigment of the macula. Like other entoptic phenomena; the bluish pattern fades rapidly if a fixed region of the retina is stimulated and so in this instrument the polarizer is made to rotate continuously.

In order to appreciate the significance of our precise measurement of eccentric fixation, which we describe later, a brief résumé of Cüppers's method may be helpful. It is essential to analyse the prognosis of a case of eccentric fixation carefully before embarking on the treatment. The success of the treatment does not depend on the estimation of the visual acuity and the type of fixation only, as many other factors have to be taken into account. To ensure a perfect and enduring result the possibilities of attaining either binocular single vision or definite alternation should be envisaged.

\section{Diagnosis and Prognosis}

Depending on the age of the patient, the prognosis may be determined by the following procedures.

Cüppers considers that the point of fixation is shown by the retinal point which bears the principal visual direction according to the results of the various investigations in each particular case. The eccentric fixation is not necessarily the point used for fixing the star of the Visuscope as this may be a point on the border of a scotoma. This is clearly demonstrated in patients who have previously had the fixing eye occluded and who fix eccentrically in this way because for them this point on the retina has a higher resolving power.

Cüppers insists on this definition because otherwise, during treatment, when use is made of monocular diplopia, the attention will be drawn to the wrong image. The localization of the principal visual direction may differ 
according as to whether the test is carried out with an after-image, Haidinger's brushes, or the star of the Visuscope. Of the tests mentioned, the star of the Visuscope resembles most nearly the fixation of an object in free space. Furthermore, Cüppers holds the opinion that the defects arising from eccentric fixation are similar to those found in anomalous retinal correspondence. It has been demonstrated that the eccentric fixation either coincides with or is less than the angle of anomaly, but is never greater.

The diagnosis of eccentric fixation and anomalous retinal correspondence may be determined by the results of the following tests:

(a) Analysis of Fixation, using

(1) The Visuscope;

(2) The after-image created by the Euthyscope;

(3) Haidinger's brushes in the Co-ordinator.

(b) Extent of Inhibition, by

(1) A comparison of the cortical and angular visual acuity†;

(2) The intensity and extent of the scotoma;

(3) The appreciation of the after-image.

(c) Bi-retinal Correspondence, by

(1) The spatial projection of the two after-images created at the maculae of the two eyes;

(2) Measurement of the objective and subjective angles of strabismus using the Maddox scale with an after-image before the non-fixing eye and a special dark red filter before the fixing eye;

(3) The Visuscope in a binocular examination;

(4) The major amblyoscope-measuring

(i) The objective and subjective angles by use of after-images, Haidinger's brushes, and real objects;

(ii) The density and extent of the suppression by use of the Bjerrum screen.

\section{Therapy}

Treatment, based on conclusions drawn from the preceding examinations, may be carried out as follows. It must be remembered that differentiation is difficult as all the symptoms are intimately connected.

†(1) The term "angular visual acuity" indicates the minimum separable measured by a single optotype. The Landolt ring, which is a test rather of the light than the form sense, is less efficient than an " $E$ " for this purpose.

(2) The term "cortical visual acuity" has been used for want of a better. It indicates a combination of the difficulties of separation in any meridian of a single row of optotypes and the disturbance caused by visual agnosia. In other words, it consists in part of the ability to recognize a complicated form and in part of discriminating it from its background. It can be measured rudimentarily by associated optotypes, no other known test existing for its complete determination.

(3) It has been noticed that in many cases of amblyopia ex anopsia, particularly in those of eccentric fixation, the angular visual acuity may be higher than the cortical visual acuity. 
(1) Attainment of foveal fixation and its principal visual direction, by means of:

(a) After-image created by the Euthyscope;

(b) Haidinger's brushes created in the Co-ordinator.

(2) Motor co-ordination of the eye and the extremities (especially the hand) when foveal fixation is assured by:

(a) After-image created by the Euthyscope using the hand;

(b) Haidinger's brushes using the hand.

(3) Elimination of inhibition when foveal fixation is assured by:

(a) After-image created by the Euthyscope;

(b) Major amblyoscope.

(4a) Elimination of difficulties of separation, which arise from rivalry of the spatial values monocularly and binocularly by:

(a) After-image and Haidinger's brushes combined with real objects projected in space and on the major amblyoscope;

(b) Attainment of binocular single vision with fusion amplitude.

(4b) Disturbances of the cerebral cortex (Gestalt Auffassung) by:

(a) Bangerter's pleoptic methods;

(b) Attainment of binocular single vision with fusion amplitude.

\section{New Method}

During our experiments with Cüppers's methods it soon became evident that it would be an advantage if we could find a method which would determine the exact position in degrees of an eccentric fixation. Initially we depicted eccentric fixation in our notes by marking approximately the position of the Visuscope star on a conventional stamped drawing of the fundus. We decided that any method we adopted must allow us to measure the relationship between three points on the fundus:

(a) The centre of the macula;

(b) The eccentric fixation point;

(c) The centre of the blind spot.

It would also be convenient if we continued to use the stamped outline of the fundus, which was so essential for marking the result of the usual Visuscope examination. These diagrams could then be given precise measurements and angles when necessary. Our method was to use the Two-Metre Bjerrum Screen and to measure the distances and angles between the points in degrees with a rotary tangent rule (Sinclair's Rule for the TwoMetre Screen).

We seated the patient on an adjusted chair before the screen, with his chin fitted firmly yet comfortably in the chin-rest opposite the fixation spot in the centre of the screen at exactly 2 metres. First we occluded the eccentric fixing eye and carefully plotted the blind spot of the normal fixing 
eye; using the rotary tangent rule we measured the angle between the fixing macula and the centre of this normal blind spot. Then, covering the normal fixing eye, the patient looked at the fixation target with his eccentrically fixing eye. We then again charted the blind spot and also plotted the scotoma due to the non-functioning macula, measuring with the scale and rule the distances and angles between the three points (centre of the blind spot, centre of the macula scotoma, and the fixation spot). We found it necessary to examine each child several times depending on his ability to co-operate. With younger children it was always easier if one of us sat near the child to give encouragement during the examination and to make sure that the fixation on the fixation spot was steady, while the other worker moved the targets and inserted the pins in the Bjerrum screen. Provided the child grasped what was required and we were able to mark out the blind spot of the sound eye, a satisfactory plot was also obtainable with the other eye, even in children as young as 5 years old.

In our calculations we assumed that the angle between the macula and the centre of the blind spot was similar in right and left eyes of the same patient. It is true that the relationship of the blind spot and the macula are affected by the Angle Alpha, but this itself is rarely asymmetrical. We have checked on the Bjerrum screen the angle between disc and macula in a number of patients who had normal fixation, including some with large degrees of anisometropia both hypermetropic and myopic, and the difference was usually less than $1^{\circ}$. Traquair (1949) states that "The distance of the blind spot from the fixation point is increased in hypermetropia and diminished in myopia in accordance with the difference in the length of the eye". It is of course agreed that, if the length of the eye only was increased or diminished in myopia and hypermetropia, our assumption and calculation would be proportionately incorrect as the anisometropia varied. Many cases do not show purely axial anomalies, and in patients who have normal eyes except for refractive error and in whom the size of the two eyes varies, the increase or decrease in length is accompanied by increase and decrease in diameter of the globe, so that the whole eye is larger or smaller. Differences in the diameter of the globe vary the distance of the blind spot from the fixation point contrary to similar alterations in length. Therefore, in by far the greater number of cases, we can assume that the angle between the macula and the centre of the blind spot is similar in the two eyes of the same patient.

\section{Case Reports}

Case 1, a boy aged 6. Visual acuity $6 / 9$ in the right eye with $+3 \mathrm{D}$ sph., and 3/60 (angular and cortical) in the left eye with $+5 \mathrm{D}$ sph.

(i) Synoptophore.-Left convergent strabismus fixing right eye objective angle $+10^{\circ}$, subjective $+4^{\circ}$.

(ii) Visuscope and Maddox Scale.-Angle of anomaly fixing right eye $+2 \triangle L / R 4 \triangle$.

(iii) After-Image and Maddox Scale.-Fixing right eye $+6^{\circ}$ objectively $0 \triangle$ L/R $4 \triangle$ subjectively. 
Both eyes were dilated with Cyclogyl 1.0 per cent. and the fixation of each eye when viewed with the Visuscope showed a normal fixation at the macula for the right eye (Fig. 1A) and an eccentric fixation for the left eye, situated superior and nasal to the macula (Fig. 1B).

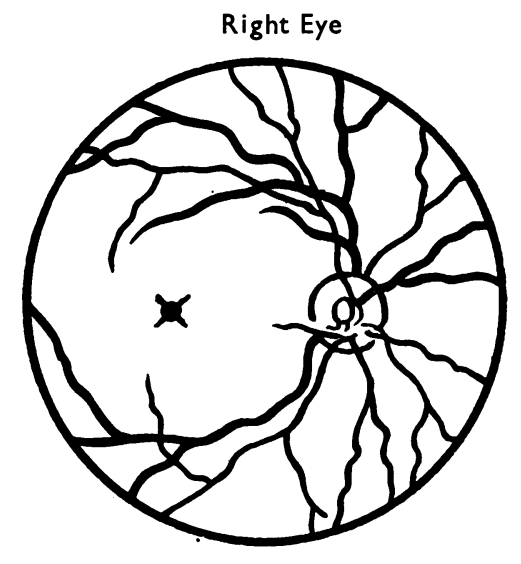

$1 \mathrm{~A}$

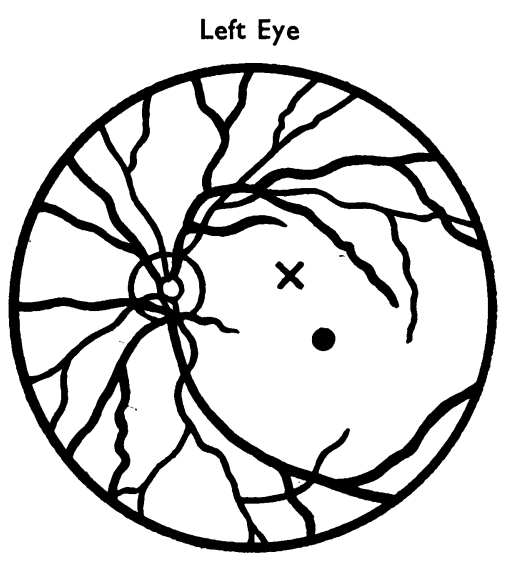

1B

Fig. 1.-Normal right eye and affected left eye, showing fixation points as seen with the Visuscope.

On the following day, when the patient's pupils had contracted, we plotted the blind spot of the right eye on the Bjerrum screen. As the child was only 6 years old we had to do this several times before we obtained full co-operation. Using the rotary tangent rule, we measured the angle between the fixing macula and the centre of the normal blind spot which was $16 \cdot 5^{\circ}$. The axis at which this angle struck the screen was slightly below the horizontal. Applying the standard notation, this was on axis $170^{\circ}$ produced below the horizontal. This we have called minus $170^{\circ}\left(-170^{\circ}\right)$. (Fig. 2A).

Covering the right eye we were able to chart the blind spot of the left eccentrically fixing eye, and we found and plotted the scotoma formed by the non-functioning macula. Using the same measurements as with the right eye, we measured the angle on the tangent scale between the fixation spot and the centre of the blind spot, which was $10.5^{\circ}$, axis $180^{\circ}$. The angle between the centre of the macular scotoma and the centre of the blind spot was $16 \cdot 5^{\circ}$. The axis at which this angle struck the screen was $-20^{\circ}$. This was measured by placing the centre of the rotary scale at the centre of the macular scotoma and noting the angle of depression between the rule and the centre of the blind spot. It will be noticed (Fig. 2B) that the angle between the centre of the blind spot and the fixing macula of the right eye is the same as that between the centre of the blind spot and the centre of the macular scotoma of the left eye, $16 \cdot 5^{\circ}$, axis $-20^{\circ}$.

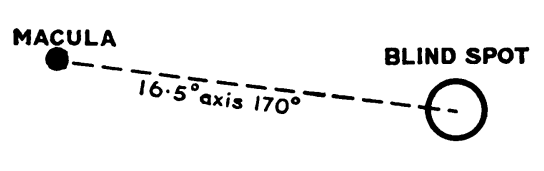

A

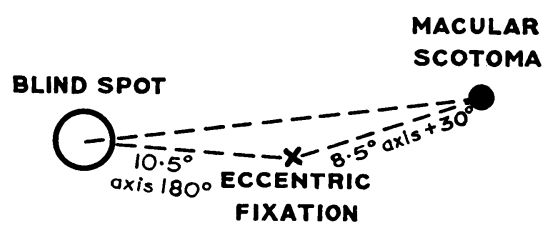

B

Fig. 2.-Normal right eye (A) and affected left eye (B) showing eccentric fixation of left eye as shown on Bjerrum screen. 
To transfer our findings to the stamped outline of the fundus it is necessary to imagine that our patient was able to focus the fixation spot with the left macula. Fig. 3 would then demonstrate the projected positions of our three points (the eccentric fixation, the macula, and the centre of the blind spot).

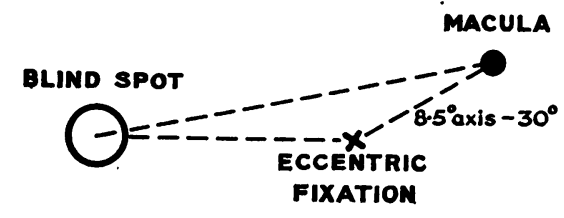

Fig. 3.-Eccentric fixation of left eye in space as shown on the Bjerrum screen.

The angle between the macular scotoma and the eccentric fixation (Fig. 2B) was $8.5^{\circ}$, axis $+30^{\circ}$; it follows that, when the macula was in a normal primary position in the centre of the fundus diagram, the eccentric fixation would still be $8.5^{\circ}$ distant from the macula, but its position on the axis would be moved from $+30^{\circ}$ to $-30^{\circ}$ (Fig. 3). In Fig. 2B the angle between the macular scotoma and the blind spot was $16.5^{\circ}$, axis $-20^{\circ}$. When this axis $-20^{\circ}$ is applied to Fig. 3 and compared with the same angle and axis of the right eye (Fig. 2A), the blind spot of the left eye is $10^{\circ}$ lower than that of the right eye.

We have measured many cases and in only a few have we noted a slight torsion of the eccentric eye when fixing. In this case we made a careful examination with the Euthyscope and we were of the opinion that when the left eye was fixing, the disc was a little higher in relation to its macula than when the right eye was examined.

In Fig. 1A and Fig. 1B the stamped diagrams of the fundi assumed the regular pattern of the macula and disc. Therefore it was necessary to readjust the axes in the few cases in which torsion of the eccentric fixing eye was demonstrated. In this particular case, the torsion was $10^{\circ}$. Any adjustment of the disc and macular angle axis would need a similar movement of the macular eccentric fixation axis so that our three point measurements remained in the same relationship. In most of the cases we examined, there was no torsion, and the diagrams of Fig. 2B moved to Fig. 3 merely by changing the signs of the axes at which these angles subtended from the eye. Therefore the final position of the angle between fixation and the macula of this left eye, in space, was $8 \cdot 5^{\circ}$, axis $-20^{\circ}$, and the blind spot macula was at $16 \cdot 5^{\circ}$, axis $-10^{\circ}$ (Fig. 4).

We have plotted the macular scotoma in space on the Bjerrum screen and we have demonstrated the position of the eccentric fixation in relation to this macula. We must now imagine the position of these points on the fundus. Any point below and nasal to the macula projects itself above and temporally. This complete reversal of points only applies when both the fundus and the screen are viewed from the same direction; in this case as viewed by the patient in space (Fig. 4), and as these points appear on the patient's fundus (Fig. 5).

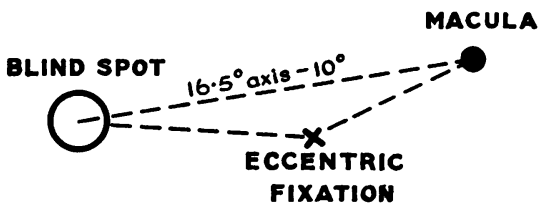

Fig. 4.-Left eye in space.

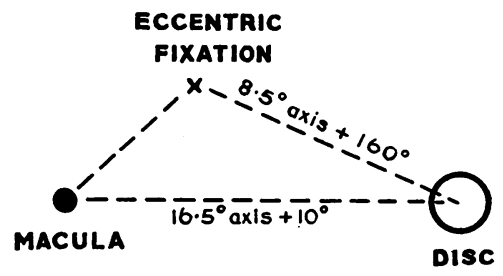

FIG. 5.-Eccentric fixation point as it appears on the patient's fundus. 
When observed, as from the patient's point of view, the position of the eccentric fixation on the fundus with the eye in its normal position, because of the reversal of points in space as on the fundus, was in the same relationship as the macular scotoma in space with the eye fixing with its eccentric fixation. Therefore the macular scotoma as demonstrated on the Bjerrum screen also demonstrates the position of the eccentric fixation on the fundus, both viewed as by the patient. It is, however, the usual practice to show the fundus as the observer sees it and to show field defects as the patient sees them. As Fig. 5 shows the positions of the blind spot and the eccentric fixation from the patient's viewpoint, then these positions must be laterally reversed to conform with the established practice of presenting fundus pictures as seen directly by the observer. The diagram (Fig. 6) shows the three positions, assumed in the stamped outline of the fundus.

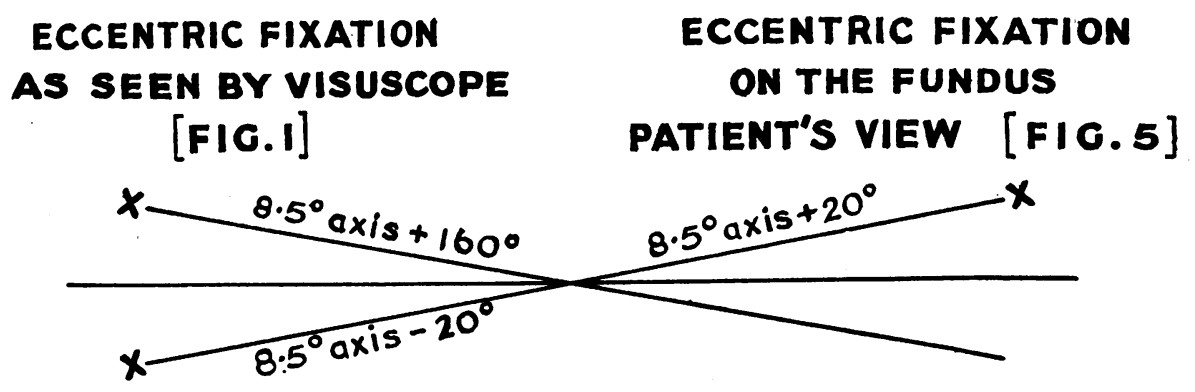

\section{ECCENTRIC FIXATION}

IN SPACE

[FICS 2,3, and 4] FIG. 6.- The three eccentric points shown in

Therefore, if the macular scotoma is plotted on the Bjerrum screen, this position laterally reversed is the position of the eccentric fixation as viewed by the Visuscope. In our illustration the macular scotoma was $8.5^{\circ}$, axis $+20^{\circ}$, and laterally reversed this makes the eccentric fixation on the fundus as seen by the Visuscope $8.5^{\circ}$, axis $+160^{\circ}$.

On occasions the macular scotoma was quite large and it was more convenient to measure the difference in the angles between the fixation and the centre of the blind spot in each eye. In practice this was found to be a quick and accurate method of measuring both large and small degrees of eccentric fixation. A small degree of eccentric fixation was effectively demonstrated in Case 2.

Case 2, a man aged 29, had had his left (good) eye excised after a motor accident 6 months before starting treatment.

Refraction.-Visual acuity in the right eye with $-3 \mathrm{D}$ sph., $-2.5 \mathrm{D}$ cyl., axis $15^{\circ}$, was $5 / 60$.

He showed an eccentric fixation with the Visuscope slightly nasal and inferior to the fovea.

Treatment.-Cüppers's method was used and the visual acuity of the right eye improved to 6/36 with foveal fixation of the Visuscope star. Monocular diplopia was appreciated 
after 6 weeks' treatment, the visual acuity of the image to the left eye being $6 / 36$ and that to the right $4 / 60$.

It was decided to measure the separation of these two images and to try to establish on which image the patient must now concentrate. It was assumed that the remaining eye was anatomically normal and the angle between the $6 / 36$ fixation and the centre of the blind spot on the Bjerrum screen was compared with the angle between the $4 / 60$ fixation and the centre of the blind spot. The angle with the 4/60 fixation coincided with the measurement of a normal adult eye. The difference between the two fixations as measured by the 2 -metre Bjerrum screen was $3^{\circ}$, and we were thus able to confirm that the image seen to the right was the image of the principal foveal direction in space.

A further example of the diagnosis of small degrees of eccentric spatial localization was found in the following case.

Case 3, a girl aged 6 years, had angular visual acuity of 12, and cortical visual acuity of $6 / 24$ in the right eye with $+1 \mathrm{D}$ sph., and visual acuity $6 / 6$ in the left eye with $+0.5 \mathrm{D}$ sph.

Between the ages of 2 and 6 years she had received intermittent total and partial occlusion of the left eye. On diagnosis with the Visuscope the right fixation was found to be juxta-foveolar. Localization of Haidinger's brushes and an after-image appeared to be central. Binocularly on the synoptophore she showed an unharmonious anomalous retinal correspondence with an angle of anomaly of $5^{\circ}$, but in space at 2 metres it was harmonious.

Treatment.-This was attempted by Cüppers's method, monocularly and binocularly, and although the visual acuity in the right eye improved to $6 / 18$ with foveolar fixation to the Visuscope star, the anomalous retinal correspondence remained intractable. Alternation was not achieved even after 3 months of total occlusion of the fixing eye. On examination of the central field a 1 to $2^{\circ}$ eccentricity was discovered in the spatial localization of the right eye.

In conclusion, it is suggested that, by the use of the Bjerrum screen, it is possible to determine the precise degree of the eccentricity in space, and that this enables small degrees of eccentric localization (undiscoverable by any other means) to be identified.

At the beginning of this paper, we pointed out the difficulties attendant on various symptoms arising from eccentric fixation and their diagnosis. This added subjective examination may help in many cases to clarify the diagnosis and the possibilities of therapy. In measuring the macular scotoma by using known sizes of scotoma targets, large and small, it is possible to demonstrate the extent of the inhibition. Definition of the eccentric fixation in degrees keeps the notes compact and is convenient for transferring the details of the case to another clinic if required.

\section{Summary}

Until Cüppers invented the Visuscope, which projects a black star on to the area of retina used by the patient to fix it, eccentric fixation was identified by the synoptophore and believed to be rare in monocular amblyopia. 
As occlusion of the better eye produces no improvement in an eye with eccentric fixation, the discovery that eccentric fixation was relatively frequent was of great practical importance.

The authors present an alternative method of detecting eccentric fixation by scotometry, which they believe to be more accurate than visuscopy though less easy to apply. Lateral reversal of the scotoma chart of the affected eye reproduces the position of the eccentric fixation as determined by visuscopy on a fundus drawing.

Assuming that the eyes are automatically symmetrical, as appears to be justified in most cases, comparison of the co-ordinates of the centre of the blind spot and the fixation point in the two eyes, suggests that monocular amblyopia is rare without some degree of eccentric fixation.

We should like to thank Mr. G. W. Black and Mr. John Foster of Leeds for permission to describe their cases.

\section{REFERENCE}

Traquair, H. M. (1949). “Clinical Perimetry”, 2nd. ed., p. 12. Kimpton, London. 